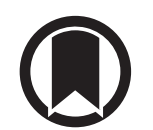

CrossMark

\title{
Anti-tumour effect of low molecular weight heparin in localised lung cancer: a phase III clinical trial
}

\author{
Guy Meyer ${ }^{1,2,3,4}$, Benjamin Besse ${ }^{5,6}$, Hélène Doubre ${ }^{7}$, Anaïs Charles-Nelson ${ }^{1}$, \\ Sandro Aquilanti ${ }^{8}$, Armine Izadifar $^{9}$, Reza Azarian ${ }^{10}$, Isabelle Monnet ${ }^{11}$, \\ Corinne Lamour ${ }^{12,13}$, Renaud Descourt ${ }^{14}$, Gérard Oliviero ${ }^{15}$, Laurent Taillade ${ }^{16}$, \\ Christos Chouaid $^{11}$. Frederique Giraud ${ }^{17}$. Pierre-Emmanuel Falcoz ${ }^{18}$, \\ Marie-Pierre Revel ${ }^{2,17}$, Virginie Westeel ${ }^{19}$, Adrien Dixmier ${ }^{20}$, Jean Tredaniel ${ }^{2,21}$, \\ Stéphanie Dehette ${ }^{22}$, Chantal Decroisette ${ }^{23}$, Alain Prevost ${ }^{24}$, Eric Pichon ${ }^{25}$, \\ Elizabeth Fabre ${ }^{1}$, Jean-Charles Soria ${ }^{5,6}$, Sylvie Friard ${ }^{7}$, Jean-Baptiste Stern ${ }^{26}$, \\ Laurence Jabot $^{11}$, Georges Dennewald ${ }^{9}$, Gérard Pavy $^{8}$, Patrick Petitpretz ${ }^{10}$, \\ Jean-Marc Tourani ${ }^{12,13}$, Marco Alifano ${ }^{2,17}$, Gilles Chatellier ${ }^{1,2,3}$ and Philippe Girard ${ }^{26}$
}

@ERSpublications

Whether the antimetastatic properties of heparins benefit patients with early-stage cancer is unknown. In this phase III trial, adjuvant tinzaparin in patients with resected lung cancer had no impact on overall survival and tumour recurrence. http://ow.ly/RZt030lpCuQ

Cite this article as: Meyer G, Besse B, Doubre H, et al. Anti-tumour effect of low molecular weight heparin in localised lung cancer: a phase III clinical trial. Eur Respir J 2018; 52: 1801220 [https://doi.org/10.1183/ 13993003.01220-2018].

ABSTRACT The anti-tumour and anti-metastatic properties of heparins have not been tested in patients with early stage cancer. Whether adjuvant low molecular weight heparin (LMWH) tinzaparin impacts the survival of patients with resected non-small cell lung cancer (NSCLC) was investigated.

Patients with completely resected stage I, II or IIIA NSCLC were randomly allocated to receive subcutaneous tinzaparin $100 \mathrm{IU} \cdot \mathrm{kg}^{-1}$ once a day for 12 weeks or no treatment in addition to standard of care. The trial was open-label with blinded central adjudication of study outcomes. The primary outcome was overall survival.

In 549 patients randomised to tinzaparin $(n=269)$ or control $(n=280)$, mean \pm sD age was $61.6 \pm 8.9$ years, 190 (34.6\%) patients had stage II-III disease, and 220 (40.1\%) patients received adjuvant chemotherapy. Median follow-up was 5.7 years. There was no significant difference in overall survival between groups (hazard ratio (HR) 1.24, 95\% CI 0.92-1.68; $\mathrm{p}=0.17$ ). There was no difference in the cumulative incidence of recurrence between groups (subdistribution HR 0.94, 95\% CI 0.68-1.30; $\mathrm{p}=0.70$ ).

Adjuvant tinzaparin had no detectable impact on overall and recurrence-free survival of patients with completely resected stage I-IIIA NSCLC. These results do not support further clinical evaluation of LMWHs as anti-tumour agents.

Published online October 4, 2018; republished October 10, 2018 with amendments to the conflict of interest disclosure statement.

This article has supplementary material available from erj.ersjournals.com

Presented in part during the XXVI meeting of the International Society on Thrombosis and Haemostasis, in Berlin, July $12,2017$.

This study is registered at ClinicalTrials.gov with identifier number NCT00475098. Requests for individual de-identified participant data, study protocol and statistical analysis plan should be sent to G. Meyer (guy.meyer@aphp.fr) or G. Chatellier (gilles.chatellier@aphp.fr).

Received: June 302018 | Accepted after revision: Aug 062018

Copyright $\odot$ ERS 2018 
Affiliations: ${ }^{1}$ Hôpital Européen Georges Pompidou, AP-HP, Paris, France. ${ }^{2}$ Université Paris Descartes, Sorbonne Paris Cité, Paris, France. ${ }^{3}$ INSERM U 970 and CIC 1418, Paris, France. ${ }^{4}$ INNOVTE, Saint Etienne, France. ${ }^{5}$ Institut Gustave Roussy, Villejuif, France. ${ }^{6}$ Université Paris Sud, Le Kremlin Bicetre, France. ${ }^{7}$ Hôpital Foch, Suresnes, France. ${ }^{8}$ Hôpital privé Arras les Bonettes, Arras, France. ${ }^{9}$ Centre Cardiologique du Nord, Saint Denis, France. ${ }^{10}$ Hôpital André Mignot, Versailles, France. ${ }^{11}$ Centre Hospitalier Intercommunal de Créteil, Créteil, France. ${ }^{12} \mathrm{CHU}$ de Poitiers, Poitiers, France. ${ }^{13}$ Université de Poitiers, Poitiers, France. ${ }^{14}$ Hôpital Morvan, $\mathrm{CHU}$ de Brest, Brest, France. ${ }^{15} \mathrm{Hôpital}$ de Longjumeau, Longjumeau, France. ${ }^{16} \mathrm{Hôpital}$ de la Pitié Salpétrière, AP-HP, Paris, France. ${ }^{17}$ Hôpital Cochin, AP-HP, Paris, France. ${ }^{18} \mathrm{CHU}$ de Strasbourg, Strasbourg, France. ${ }^{19} \mathrm{CHU}$ de Besançon, Besançon, France. ${ }^{20}$ Centre hospitalier régional d'Orléans, Orléans, France. ${ }^{21}$ Hopital Saint-Joseph, Paris, France. ${ }^{22}$ Centre hospitalier de Compiègne, Compiègne, France. ${ }^{23}$ Centre hospitalier régional d'Annecy, Annecy, France. ${ }^{24}$ Institut Jean Godinot, Reims, France. ${ }^{25} \mathrm{CHU}$ de Tours, hôpital Bretonneux, Tours, France. ${ }^{26}$ Institut du Thorax Curie-Montsouris, Institut Mutualiste Montsouris, Paris, France.

Correspondence: Philippe Girard, Institut du Thorax Curie-Montsouris, Institut Mutualiste Montsouris, 42 boulevard Jourdan, 75014, Paris, France. E-mail: philippe.girarddimm.fr

\section{Introduction}

The interactions between coagulation and cancer progression as well as the anti-tumour properties of heparins are widely documented in vitro and in animal studies [1-6]. In particular, P-selectin inhibition, the reduction in endothelial adhesion, and anti-angiogenesis are the main mechanisms through which heparins reduce the seeding and early growth of micrometastases. In contrast, the results of the few studies that specifically addressed the effect of heparins on the survival of cancer patients are conflicting and mostly negative [7-12]. However, not only were most of these studies conducted in patients with various types of cancers, making their results difficult to apply to one specific cancer type $[8,10,13]$, but also all studies included a majority of patients with locally advanced or metastatic disease, i.e. patients for whom inhibiting the early stages of the metastatic process appears of little relevance. Actually, subgroup analyses of previous trials and a post hoc analysis of a randomised trial of patients with cancer-associated thrombosis suggest that the effect of heparins on survival may be more pronounced in, or limited to, patients with early stage cancers $[9,11,14]$, but this question has not been addressed specifically and additional research has been encouraged $[13,15]$.

Lung cancer is the leading cause of death from cancer worldwide. Non-small cell lung cancers (NSCLCs) represent about $80 \%$ of lung cancers. Even in patients with early stage resectable NSCLC, the 5 -year overall survival remains around $60 \%[16,17]$. The Tinzaparin in Lung Tumors (TILT) study was designed to prospectively assess whether low molecular weight heparin (LMWH) tinzaparin could improve overall survival in patients with resected stage I-III NSCLC.

\section{Methods \\ Study design and oversight}

The TILT trial is a randomised, multicentre, open, controlled trial with blinded adjudication of outcomes. The trial was conducted in accordance with the Declaration of Helsinki and Good Clinical Practice guidelines and was approved by the Independent Ethics Committee Ile-de-France I, France. Written informed consent was obtained from all patients. The study was designed and supervised by an executive steering committee and was sponsored by Assistance Publique-Hôpitaux de Paris, which had no role in the decision to submit the manuscript for publication. An independent data and safety monitoring committee reviewed the safety data. All the authors had full access to the data and vouch for the accuracy and completeness of the data and attest that the trial was conducted in accordance with the protocol and all amendments. This trial is registered with ClinicalTrials.gov, number NCT00475098.

\section{Patients}

Patients aged over 18 years with completely resected stage I, II or IIIA NSCLC were included within 8 weeks of surgery. Detailed inclusion and exclusion criteria are provided in the supplementary appendix. Stage classification was made according to the TNM staging in use at the time of inclusion, i.e. the sixth edition until December 2008 and the seventh edition thereafter $[16,18]$.

\section{Randomisation}

Eligible patients were randomly assigned in a 1:1 ratio to receive either tinzaparin or no anticoagulants according to a list of randomisation numbers with treatment assignments. This list was computer-generated, used alternate blocks of small size $(2,4,6)$ to make it unpredictable and was stratified according to centre and tumour stage (I versus II-III). An internet application (CleanWeb) allowed central randomisation. 


\section{Interventions}

In the experimental arm, tinzaparin (Innohep; Leo Pharma France, Vernouillet, France) was administered at a dose of $100 \mathrm{IU} \cdot \mathrm{kg}^{-1}$ subcutaneously, once daily on top of usual care, for 12 weeks. The treatment was started as soon as possible after the inclusion visit and less than 8 weeks after surgery. It was stopped in patients with either a platelet count of less than 30000 per $\mathrm{mm}^{3}$ or suspected heparin-induced thrombocytopenia.

\section{Usual care}

In both groups, the decision to administer adjuvant chemotherapy was made during routine multidisciplinary meetings at each centre. The decision depended mainly on pathological stage, performance status, comorbidities and patient preferences, and was made independently of any proposal to participate in the study. In patients who were given chemotherapy, platinum-based (preferably cis-platinum) doublets for three or four cycles were recommended [19].

\section{Patient management and follow-up}

During the 12-week study treatment period, two additional visits specific to the trial were scheduled at 4 and 8 weeks after the start of study treatment in the tinzaparin group. These visits were optional in the control group. A 16-week post-randomisation visit was scheduled in both groups. Afterwards, at least one visit was scheduled each year in both groups, until the end of the study. When a patient did not attend a visit, every effort was made to contact the patient by the local investigator. When it was not possible to obtain relevant information, the patient's vital status was obtained from birth and death registry of the patient's birthplace.

\section{Outcomes}

The study primary outcome was overall survival. Secondary outcome measures included serious bleeding recorded during the 12-week treatment period in the tinzaparin group or the corresponding period in the control group (supplementary appendix), recurrence-free survival, cancer-related mortality, and symptomatic venous thromboembolic events recorded during the whole follow-up period (supplementary appendix). All suspected outcome events and deaths were adjudicated by an independent clinical events committee whose members were unaware of treatment assignment.

\section{Sample size}

The sample size estimate for the primary efficacy outcome was based on the anticipated mortality rate of patients with completely resected stage I, II or IIIA NSCLC, estimated at $10 \%$ per year in the control group [17]. To detect a hazard ratio (HR) of 0.66 , with an $80 \%$ power, a $5 \%$ type 1 error and a 3 -year follow-up for each patient, inclusion of 800 patients was needed.

In June 2010, due to a slower than expected inclusion rate, and without knowledge of the events rate, the decision was made to reassess the sample size. We decided to follow all patients up to a fixed end-of-study date which was set at 3 years after inclusion of the last patient rather than a fixed duration of 3 years for each patient. According to the recruitment rate, the expected median follow-up duration increased to 4 years. By increasing the number of expected events, with the same hypotheses, to detect a hazard ratio of 0.66, inclusion of 550 patients was needed. Calculations were made using the nQuery Advisor 4.0 software.

\section{Statistical analysis}

Analyses were performed according to the intention-to-treat principle, with the use of data updated on June 15, 2016. Survival was calculated from the randomisation date to the date of death (overall survival), date of recurrence (recurrence-free survival), or the date of last contact in case of censoring. Data for patients who did not reach the primary outcome or were lost to follow-up were censored at the last known follow-up visit. The per-protocol population was defined as all patients without major deviation in the inclusion and exclusion criteria (supplementary appendix) and who received $\geqslant 30$ days of tinzaparin in the experimental group.

Time-to-event curves were constructed using the Kaplan-Meier method and were compared by the log-rank test, with hazard ratios and 95\% confidence intervals estimated with the Cox model. The proportional hazards assumption was checked using Schoenfeld residuals. For estimating the cumulative incidence of recurrence, deaths unrelated to the index lung cancer were considered as competing risk, with the use of cumulative incidence curves. Between-group comparisons were done using the Gray test and the Fine and Gray model was used to estimate the subdistribution hazard ratios (SHRs) [20]. The proportional hazards assumption for the Fine and Gray model was checked graphically [21]. Models were adjusted on the tumour stage. 
Pre-specified subgroup analyses included tumour stage (I versus II-III) and adjuvant chemotherapy (yes or no). Interaction between the treatment and stage and between treatment and adjuvant chemotherapy was tested using the Cox model to investigate whether the treatment effect on the primary outcome differed in the pre-planned subgroups of interest. All tests were two-sided. Analyses were performed with SAS software, version 9.3, or R software, version 2.14.0 (survival and cmprsk packages). Presentation of results is made in accordance with the CONSORT statement [22].

\section{Role of the funder}

The study was supported by two grants issued by the French Ministry of Health (PHRC AOM05185 and PHRC AOM12612). The funder had no role in study design, data collection, data gathering, data interpretation, or writing of the manuscript. Leo Pharma provided the study drug and a complementary grant but had no role in the study design, conduct of the study, data analysis and decision to submit the manuscript.

\section{Results \\ Patients}

From August 2007 to June 2013, 553 patients were included at 35 centres in France (supplementary appendix). Four patients withdrew their consent (three tinzaparin and one control) for both study participation and use of their data. The remaining 549 patients were included in the intention-to-treat analysis, 280 in the control group and 269 in the tinzaparin group (figure 1). Baseline demographic and disease characteristics were well balanced between the two study groups (table 1).

In the tinzaparin group, eight (3.0\%) patients did not receive any dose of study drug, an additional 19 (7.1\%) patients received $<30$ days of study drug and $224(83.3 \%)$ patients received $>8$ weeks of treatment. Median follow-up was 5.7 years (interquartile range 5.4-5.9 years). Vital status was available in all but two patients on June 15, 2016.

\section{Primary outcome}

79 and 89 patients died in the control and tinzaparin groups, respectively. Overall survival was not significantly different between the two groups (HR 1.24, 95\% CI 0.92-1.68; p=0.17) (figure 2). The 5-year

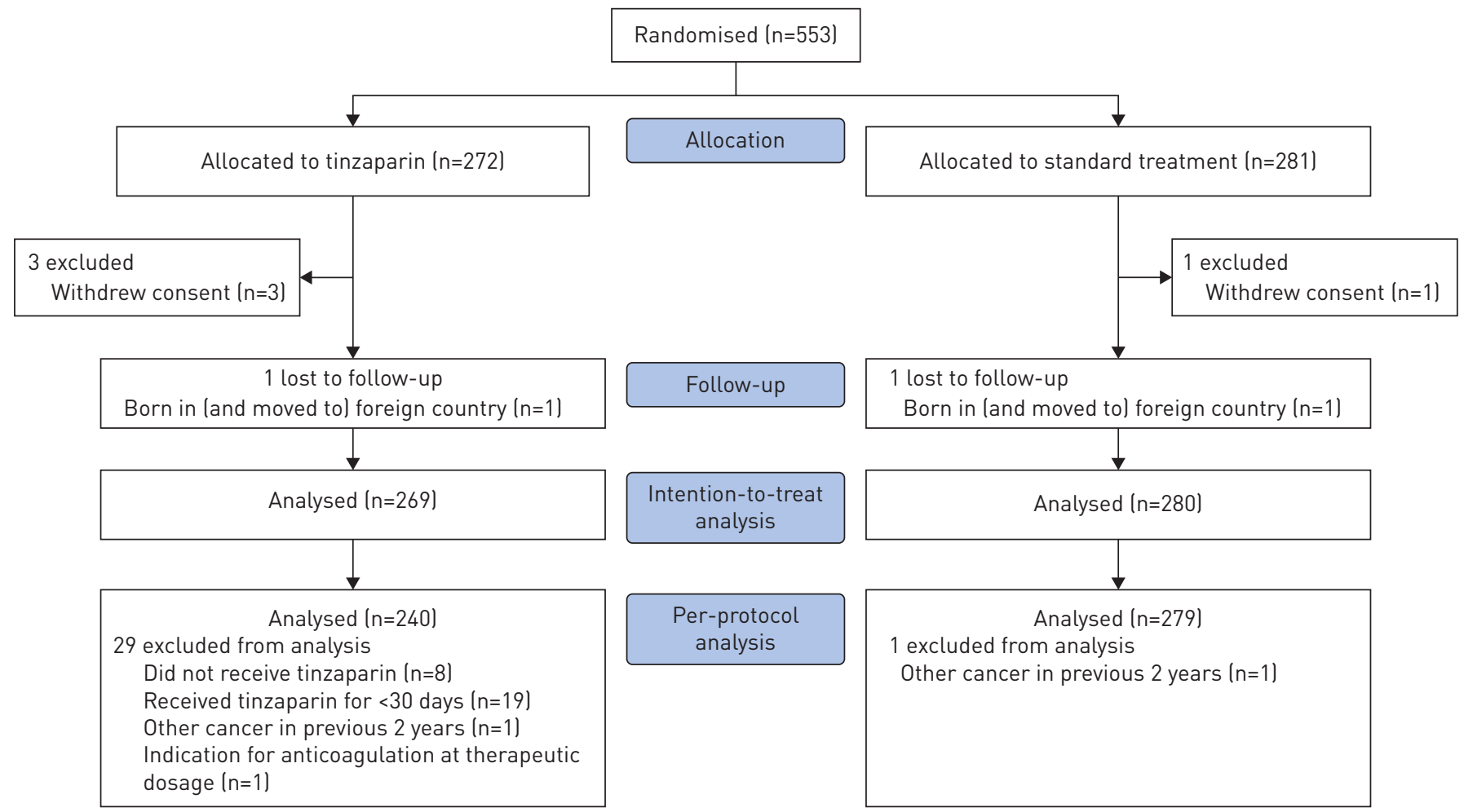

FIGURE 1 CONSORT flow diagram of trial participants. The intention-to-treat population included all the patients who had undergone randomisation minus the four patients who withdrew consent. The per-protocol population was defined as all patients without major deviation in the inclusion and exclusion criteria and who received $\geqslant 30$ days of tinzaparin in the experimental group. 
TABLE 1 Demographics and characteristics of 549 patients at baseline

\begin{tabular}{|c|c|c|}
\hline & Control $(n=280)$ & Tinzaparin $(n=269)$ \\
\hline Age years & $61.6 \pm 8.8$ & $61.6 \pm 9.0$ \\
\hline Males & $189(67.5)$ & $167(62.1)$ \\
\hline \multicolumn{3}{|l|}{ Performance status ( $n=535$ ) } \\
\hline 0 & $179(65.6)$ & $168(64.1)$ \\
\hline 1 & $93(34.1)$ & $89(34.0)$ \\
\hline 2 & $1(0.4)$ & $5(1.9)$ \\
\hline Past or current smokers & $256(91.4)$ & $239(88.8)$ \\
\hline Smoking history pack-years ( $n=497$ ) & $40(30-50)$ & $40(30-51)$ \\
\hline Body mass index $\mathrm{kg} \cdot \mathrm{m}^{-2}$ & $24.5 \pm 4.5$ & $24.9 \pm 4.9$ \\
\hline Creatinine level $\mu \mathrm{mol} \cdot \mathrm{L}^{-1}$ ( $\mathrm{n}=533$ ) & $72(60-82)$ & $71(62-84)$ \\
\hline Antiplatelet therapy $(n=544)$ & $61(21.9)$ & $62(23.4)$ \\
\hline Neo-adjuvant chemotherapy ( $n=548$ ) & $17(6.1)$ & $24(9.0)$ \\
\hline Neo-adjuvant radiotherapy $(n=548)$ & $2(0.7)$ & $0(0.0)$ \\
\hline \multicolumn{3}{|l|}{ Type of surgery } \\
\hline Segmentectomy & $17(6.1)$ & $6(2.2)$ \\
\hline Lobectomy (or bilobectomy) & $241(86.0)$ & $235(87.4)$ \\
\hline Pneumonectomy & $22(7.9)$ & $28(10.4)$ \\
\hline \multicolumn{3}{|l|}{ Histology" } \\
\hline Squamous cell carcinoma & $80(28.6)$ & $68(25.3)$ \\
\hline Adenocarcioma & $183(65.4)$ & 172 (63.9) \\
\hline Large cell carcinoma & $7(2.5)$ & $18(6.7)$ \\
\hline Others & $10(3.6)$ & $11(4.1)$ \\
\hline \multicolumn{3}{|l|}{ Cancer stage ${ }^{\pi_{1}+}$} \\
\hline Stage $0-1$ & $185(66.1)$ & $174(64.7)$ \\
\hline Stage II & $64(22.9)$ & $71(26.4)$ \\
\hline Stage III & $31(11.1)$ & $24(8.9)$ \\
\hline Duration of post-operative LMWH prophylaxis days ( $n=542$ ) & $11.72 \pm 7.78$ & $10.84 \pm 7.34$ \\
\hline Adjuvant chemotherapy & $110(39.3)$ & $110(40.9)$ \\
\hline Stage I patients & $27(9.6)$ & $35(13.0)$ \\
\hline Stage II-III patients & $83(29.6)$ & $75(27.9)$ \\
\hline Adjuvant radiotherapy & $15(5.4)$ & $16(5.9)$ \\
\hline Time from surgery to randomisation days & $37.5 \pm 13.4$ & $37.7 \pm 14.1$ \\
\hline \multicolumn{3}{|c|}{$\begin{array}{l}\text { Data are presented as mean } \pm \text { SD, } n(\%) \text { or median (interquartile range). The } 549 \text { patients represent the } \\
\text { intention-to-treat population ( } 553 \text { included patients minus four patients who withdrew consent and removal } \\
\text { of their data from the database). There were no significant differences ( } p<0.05 \text { ) between arms for any of } \\
\text { the baseline characteristics. LMWH: low molecular weight heparin. \#: one patient had two different } \\
\text { histological types in the control group; }{ }^{\uparrow}: \text { TN categories ypTN or pTN; }{ }^{+}: \text {one patient had stage ypTONO in } \\
\text { the tinzaparin group and one patient had stage IIIB in the control group. }\end{array}$} \\
\hline
\end{tabular}

overall survival was $74.2 \%$ (95\% CI 68.9\%-79.9\%) and 68.2\% (95\% CI 62.5\%-74.4\%) in the control and tinzaparin groups, respectively.

\section{Secondary outcomes}

The index lung cancer was the cause of death in $46(16.4 \%)$ and $50(18.6 \%)$ patients in the control and tinzaparin groups, respectively (SHR 1.17, 95\% CI 0.78-1.74; $\mathrm{p}=0.46$ ) (table 2). The cumulative incidence of recurrence was not significantly different between the two groups (SHR 0.94, 95\% CI 0.68-1.30; $\mathrm{p}=0.70$ ) (figure 3). The 5-year cumulative incidence of recurrence was $27.8 \%$ (95\% CI $22.4 \%-33.2 \%$ ) and $25.9 \%$ (95\% CI 20.6\%-31.3\%) in the control and tinzaparin groups, respectively. Two patients in the tinzaparin group (versus none in the control group) experienced a serious non-fatal bleeding event during the treatment period. Minor bleeding events occurred in one patient $(0.4 \%)$ and 19 patients (7.1\%) in the control and tinzaparin groups during the treatment period, respectively $(\mathrm{p}<0.001)$. Symptomatic venous thromboembolism occurred during the whole follow-up period in $20(7.1 \%)$ patients in the control group and $18(6.7 \%)$ patients in the tinzaparin group (SHR 0.95 , 95\% CI $0.68-1.32 ; \mathrm{p}=0.75$ ).

\section{Subgroup analysis}

Among the 220 patients who received adjuvant chemotherapy, the use of tinzaparin was associated with a significantly decreased overall survival (HR 1.78, 95\% CI 1.13-2.81; p for interaction=0.03) whereas no difference was observed between treatment groups among the 329 patients who did not receive adjuvant chemotherapy (figure 4). Treatment group was the only significant predictor of death in patients who 
FIGURE 2 Overall survival in the intention-to-treat population. Kaplan-Meier estimates of overall survival according to treatment group in the intention-to-treat population. Overall survival at 5 years was estimated at $68.2 \%$ $195 \% \mathrm{Cl} 62.5-74.4 \%)$ in the tinzaparin group versus $74.2 \% 195 \%$ $\mathrm{Cl} 68.9-79.9 \%)$ in the control group. The $p$-value was calculated using log-rank test. HR: hazard ratio.

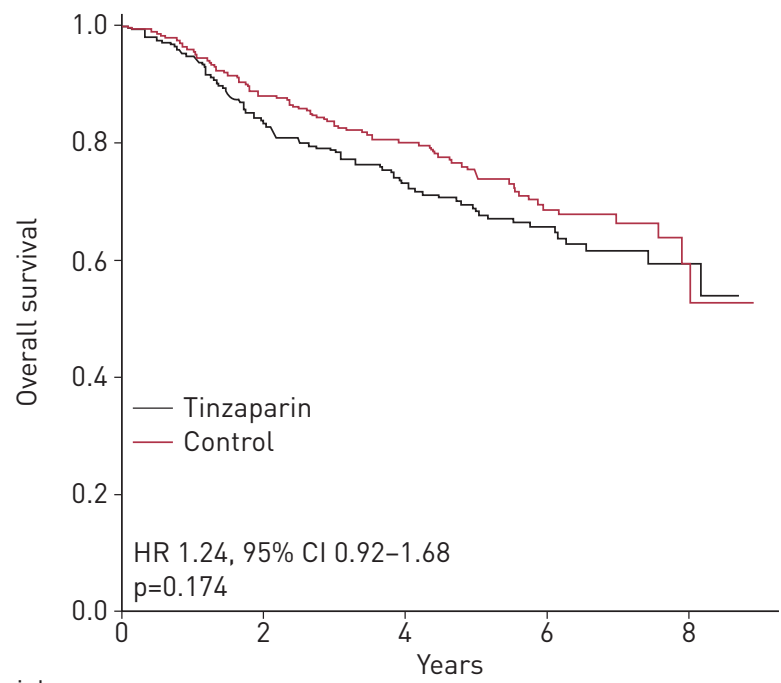

At risk $n$

Tinzaparin 269

Control 280

225

246

153

177

78

16

TABLE 2 Causes of death (clinical events committee adjudications) according to treatment arm in pre-planned subgroups analyses by pathological stage and adjuvant chemotherapy

Stage

Stage I

Index lung cancer

Other cancer

Serious bleeding

Cardiovascular disease

Respiratory disease

Other

Unknown

Stage II-III

Index lung cancer

Other cancer

Serious bleeding

Cardiovascular disease

Respiratory disease

Other

Unknown

\section{Adjuvant chemotherapy}

Yes

Index lung cancer

Other cancer

Serious bleeding

Cardiovascular disease

Respiratory disease

Other

Unknown

No

Index lung cancer

Other cancer

Serious bleeding

Cardiovascular disease

Respiratory disease

Other

Unknown
Control

$79 / 280$

47/185

Tinzaparin

$89 / 269$

$43 / 174$

21

8

2

2

1

3

6

46/95

$32 / 95$

24

1

3

4

$30 / 110$

22

1

1

4

2

$49 / 170$

24

5

2 
FIGURE 3 Cumulative risk of adjudicated recurrence of the index lung cancer in the intention-to-treat population. Death from causes other than the index lung cancer is the competing risk (dashed lines). HR: hazard ratio.

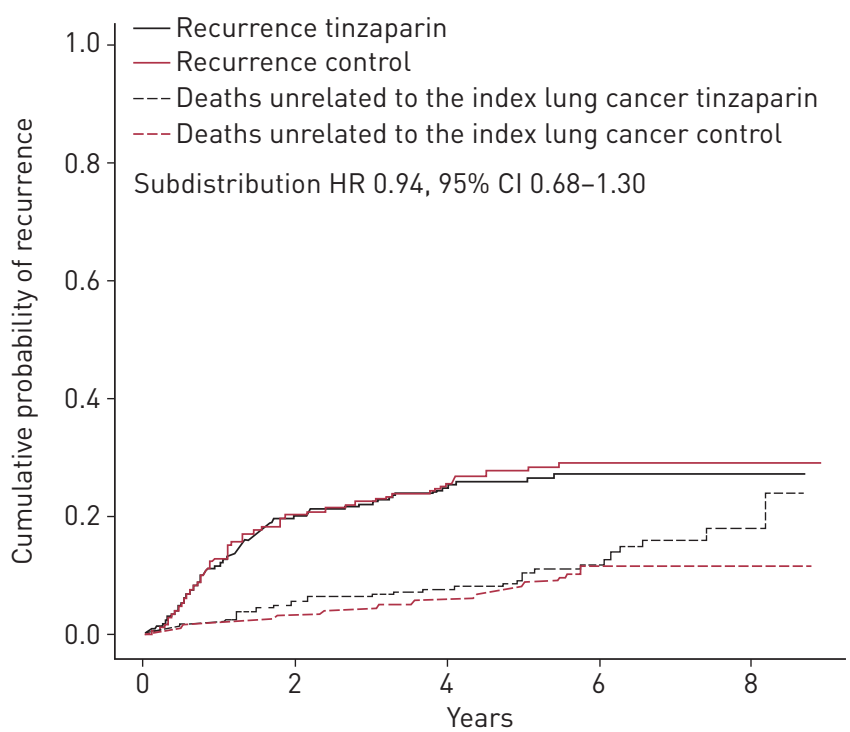

received adjuvant chemotherapy (supplementary appendix, table A1). The initial lung cancer was the cause of death in 22 patients $(20.0 \%)$ and 33 patients $(30.0 \%)$ in the control and tinzaparin groups, respectively (table 2) and the cumulative incidence of recurrence was not significantly different between the two treatment groups (SHR 1.17, 95\% CI 0.76-1.83; p=0.47) (figure 5).

Among the 190 patients with stage II-III disease, the use of tinzaparin was associated with a decreased overall survival (HR 1.61, 95\% CI 1.03-2.54; p-value for interaction 0.13), whereas no difference was observed between treatment groups among the 359 patients with stage I disease (figure 4). There were no potential predictors of death in patients with stage II-III lung cancer (supplementary appendix, table A2). The initial lung cancer was the cause of death in 24 patients $(25.3 \%)$ and 29 patients (30.5\%) in the control and tinzaparin groups, respectively (table 2) and the cumulative incidence of recurrence was not significantly different between groups (figure 5).

\section{Per-protocol analysis}

The per-protocol study population consisted of 519 patients; 279 in the control group and 240 in the tinzaparin group (figure 1). No significant difference in overall survival was observed between groups, neither in the overall population, nor in the stage I or stage II-III subgroups, but the difference remained significant in patients who received chemotherapy (supplementary appendix, figure A1). The cumulative incidence of recurrence was not significantly different between groups neither in the total per-protocol population, nor in the two subgroup analyses (supplementary appendix, figure A2). Adjustment for confounding factors did not modify these results.

\section{Discussion}

In this large multicentre randomised trial, tinzaparin given as an adjuvant therapy in addition to standard of care in patients with completely resected stage I-III NSCLC was not associated with either an improved

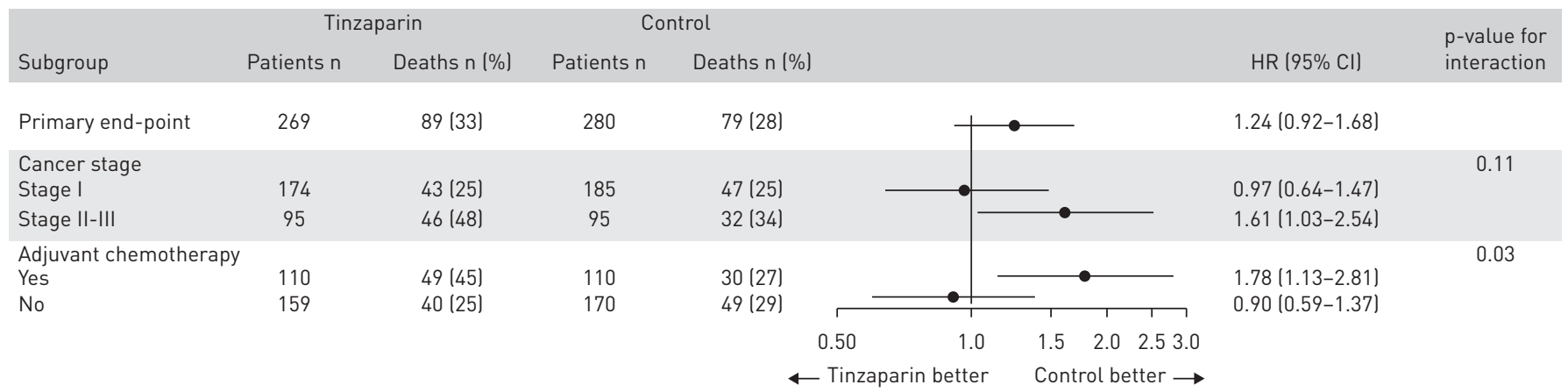

FIGURE 4 Overall survival in the two pre-planned subgroup analyses on the use of adjuvant chemotherapy and on tumour stage (I versus II-III) (intention-to treat analysis). HR: hazard ratio. 


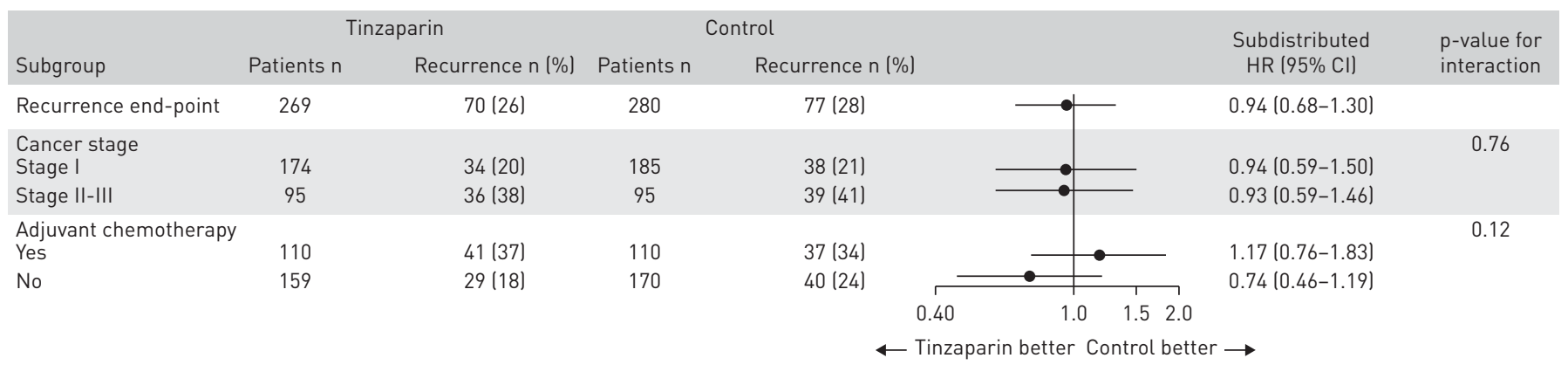

FIGURE 5 Cumulative risk of recurrence of the index lung cancer in the two pre-planned subgroup analyses on the use of adjuvant chemotherapy and on tumour stage (I versus II-III) (intention-to-treat population). Death from causes other than the index lung cancer is the competing risk. HR: hazard ratio.

overall survival or a decreased risk of tumour recurrence. The large sample size, the homogeneous study population with a single cancer type, the high compliance with the study drug, the long follow-up period with a very low rate of patients lost to follow-up and the robust primary end-point of overall survival all support the reliability of the study results.

Previous clinical trials exploring the role of heparins as anticancer agents were performed mostly or only in patients with locally advanced or metastatic disease, and therefore could not answer the question of the role of heparins in early stage cancers suggested by some experimental results. The TILT study, with its unique homogeneous population of NSCLC patients who had no detectable metastases at the time of inclusion, was designed to address this question. Combined with the negative results obtained in advanced cancer, the present results strongly advocate against any clinically significant effect of LMWH as anti-cancer treatment.

In the present trial, the treatment was to be started within 2 months of surgery and prolonged for 3 months, paralleling eventual adjuvant chemotherapy when indicated. An "intermediate" (neither prophylactic nor therapeutic) dose of tinzaparin was selected in order to parallel some experimental results suggesting a dose-response relationship of the anti-cancer effect [23], and to take into account the results from previous studies suggesting that prophylactic doses are generally not associated with an increased survival $[9,13,14]$, while avoiding the bleeding risk associated with therapeutic doses. Whether higher doses and/or longer treatment durations would have produced different findings is unknown, but the present results do not provide support for such research. Finally, in the animal models showing a reduction in the metastatic spread, heparin was administrated before the intravenous injection of tumour cells [5]. Therefore, the question of a different timing of heparin administration (e.g. starting tinzaparin sooner after, or even before, the surgery) could make sense and merit further investigation. This hypothesis is currently tested in a trial of colorectal cancer patients (NCT01455831).

The results of the pre-planned subgroup analyses by tumour stage and the use of adjuvant chemotherapy are unexpected and should be interpreted with caution in the context of a negative trial. In the analysis by tumour stage, the interaction test did not reach statistical significance, and the survival difference observed in stage II-III disease between arms was no longer significant in the per-protocol population. In patients who received adjuvant chemotherapy, of whom $71 \%$ had stage II-III tumours, the use of tinzaparin was associated with a lower survival and the difference remained significant in the per-protocol population. However, unlike stage, randomisation was not stratified on adjuvant chemotherapy, which may have resulted in unidentified imbalances between arms. No difference was observed in the cumulative risk of recurrence between arms in this subgroup, advocating against a direct negative interaction between tinzaparin and chemotherapy. In addition, in vitro experiments suggest that tinzaparin reverses cisplatin resistance of human ovarian cancer cells [24]. Thus, the reasons for the excess of deaths observed in the tinzaparin arm of adjuvant treated patients are difficult to ascertain. In any case, these subgroup analysis findings, although intriguing, can be regarded only as hypothesis-generating.

The study limitations include the open-label design, the 6 years needed to complete inclusions, the lower than expected death rate and the use of post-operative prophylactic LMWH in both groups. Regarding the open-label design, the main study outcome was overall mortality, which minimises the probability of bias, and all clinical end-points, including the cause of death, were blindly adjudicated. As for the long inclusion and follow-up periods, the 5-year survival rate remains a reliable end-point to compare cancer treatments, and the large number of patients still at risk at 5 years strengthens the study results. Also, adjuvant therapy for resected NSCLC remained unchanged over the entire inclusion and follow-up periods, making the reference treatment a valid comparator. The higher than expected survival rate is 
likely explained by the inclusion of a large proportion of stage I patients (65\%) and the use of modern staging tools, including positron emission tomography scanning and brain magnetic resonance imaging that improve staging accuracy and, artificially, prognosis [25]. Finally, the short (11 days) post-operative administration of LMWH in both groups, as per current guidelines, is unlikely to explain our negative findings.

This study could not demonstrate any clinical benefit from tinzaparin as an adjuvant treatment for resected early stage NSCLC. Combined with the negative findings of a recent trial in advanced lung cancer [13], these results suggest that, whatever the role of coagulation in cancer progression, further exploration of heparins as anti-tumour agents, at least in lung cancer, should no longer be considered a clinically relevant approach.

Acknowledgements: We thank all nice and hard-working persons of the Clinical Trial Unit of the Georges Pompidou European Hospital in charge of trial organisation, data monitoring, collection, and management.

Author contributions: G. Meyer, P. Girard and M. Alifano are the coordinating investigators of the TILT trial, they made substantial contributions to the conception and design of the trial, drafted the manuscript, and revised the manuscript critically for important intellectual content. G. Chatellier made substantial contributions to the conception and design of the trial, drafted the manuscript, and revised the manuscript critically for important intellectual content. All authors read and approved the final manuscript and agree to be accountable for all aspects of the work in ensuring that questions related to the accuracy or integrity of any part of the work are appropriately investigated and resolved.

Conflict of interest: G. Meyer reports grants and non-financial support from Leo Pharma, outside the submitted work. B. Besse has nothing to disclose. H. Doubre has nothing to disclose. A. Charles-Nelson has nothing to disclose. S. Aquilanti reports non-financial support from Leo Pharma, outside the submitted work. A. Izadifar has nothing to disclose. R. Azarian has nothing to disclose. I. Monnet has nothing to disclose. C. Lamour has nothing to disclose. R. Descourt has nothing to disclose. G. Oliviero has nothing to disclose. L. Taillade has nothing to disclose. C. Chouaid has nothing to disclose. F. Giraud has nothing to disclose. P-E. Falcoz has nothing to disclose. M-P. Revel has nothing to disclose. V. Westeel has nothing to disclose. A. Dixmier has nothing to disclose. J. Tredaniel has nothing to disclose. S. Dehette has nothing to disclose. C. Decroisette has nothing to disclose. A. Prevost has nothing to disclose. E. Pichon has nothing to disclose. E. Fabre has nothing to disclose. J-C. Soria is a full time employee at Medimmune (this position was undertaken after completion of the study), and has received consultancy fees from AstraZeneca, Roche, Sanofi, Servier, Abbvie and Pharmamar, outside the submitted work. S. Friard has nothing to disclose. J-B. Stern has nothing to disclose. L. Jabot has nothing to disclose. G. Dennewald has nothing to disclose. G. Pavy has nothing to disclose. P. Petitpretz has nothing to disclose. J-M. Tourani has nothing to disclose. M. Alifano has nothing to disclose. Dr. Chatellier has nothing to disclose. P. Girard reports personal fees and non-financial support from Leo Pharma, outside the submitted work.

Support statement: The trial was sponsored by the Assistance Publique-Hôpitaux de Paris and funded by two grants from the Programme Hospitalier de Recherche Clinique, French Ministry of Health, (PHRC AOM05185 and PHRC AOM12612). Leo Pharma provided the drug and an additional grant. Funding information for this article has been deposited with the Crossref Funder Registry.

\section{References}

1 Folkman J, Weisz PB, Joullie MM, et al. Control of angiogenesis with synthetic heparin substitutes. Science 1989; 243: $1490-1493$

2 Hejna M, Raderer M, Zielinski CC. Inhibition of metastases by anticoagulants. J Natl Cancer Inst 1999; 91: 22-36.

3 Ludwig RJ, Alban S, Bistrian R, et al. The ability of different forms of heparins to suppress P-selectin function in vitro correlates to their inhibitory capacity on bloodborne metastasis in vivo. Thromb Haemost 2006; 95: 535-540.

4 Smorenburg SM, Vink R, Otten HM, et al. The effects of vitamin K-antagonists on survival of patients with malignancy: a systematic analysis. Thromb Haemost 2001; 86: 1586-1587.

5 Stevenson JL, Choi SH, Varki A. Differential metastasis inhibition by clinically relevant levels of heparins correlation with selectin inhibition, not antithrombotic activity. Clin Cancer Res 2005; 11: 7003-7011.

6 Zacharski LR, Ornstein DL Heparin and cancer. Thromb Haemost 1998; 80: 10-23.

7 Lebeau B, Chastang C, Brechot JM, et al. Subcutaneous heparin treatment increases survival in small cell lung cancer. "Petites Cellules" Group. Cancer 1994; 74: 38-45.

8 Kakkar AK, Levine MN, Kadziola Z, et al. Low molecular weight heparin, therapy with dalteparin, and survival in advanced cancer: the fragmin advanced malignancy outcome study (FAMOUS). J Clin Oncol 2004; 22: 1944-1948.

9 Klerk CP, Smorenburg SM, Otten HM, et al. The effect of low molecular weight heparin on survival in patients with advanced malignancy. J Clin Oncol 2005; 23: 2130-2135.

10 Sideras K, Schaefer PL, Okuno SH, et al. Low-molecular-weight heparin in patients with advanced cancer: a phase 3 clinical trial. Mayo Clin Proc 2006; 81: 758-767.

11 Altinbas M, Coskun HS, Er O, et al. A randomized clinical trial of combination chemotherapy with and without low-molecular-weight heparin in small cell lung cancer. J Thromb Haemost 2004; 2: 1266-1271.

12 van Doormaal FF, Di Nisio M, Otten HM, et al. Randomized trial of the effect of the low molecular weight heparin nadroparin on survival in patients with cancer. J Clin Oncol 2011; 29: 2071-2076.

13 Macbeth F, Noble S, Evans J, et al. Randomized phase III trial of standard therapy plus low molecular weight heparin in patients with lung cancer: FRAGMATIC Trial. J Clin Oncol 2016; 34: 488-494.

14 Lee AY, Rickles FR, Julian JA, et al. Randomized comparison of low molecular weight heparin and coumarin derivatives on the survival of patients with cancer and venous thromboembolism. J Clin Oncol 2005; 23: 2123-2129. 
15 Lyman GH, Khorana AA, Kuderer NM, et al. Venous thromboembolism prophylaxis and treatment in patients with cancer: American Society of Clinical Oncology clinical practice guideline update. J Clin Oncol 2013; 31: 2189-2204.

16 Goldstraw P, Crowley J, Chansky K, et al. The IASLC Lung Cancer Staging Project: proposals for the revision of the TNM stage groupings in the forthcoming (seventh) edition of the TNM Classification of malignant tumours. J Thorac Oncol 2007; 2: 706-714.

17 Arriagada R, Bergman B, Dunant A, et al. Cisplatin-based adjuvant chemotherapy in patients with completely resected non-small-cell lung cancer. $N$ Engl J Med 2004; 350: 351-360.

18 Mountain CF. Revisions in the International System for Staging Lung Cancer. Chest 1997; 111: 1710-1717.

19 Pignon JP, Tribodet H, Scagliotti GV, et al. Lung adjuvant cisplatin evaluation: a pooled analysis by the LACE Collaborative Group. J Clin Oncol 2008; 26: 3552-3559.

20 Fine J, Gray R. A proportional hazards model for the subdistribution of a competing risk. J Am Stat Assoc 1999; 94: 496-509.

21 Liu M, Lu W, Shao Y. A Monte Carlo approach for change-point detection in the Cox proportional hazards model. Stat Med 2008; 27: 3894-3909.

22 Schulz KF, Altman DG, Moher D, et al. CONSORT 2010 statement: updated guidelines for reporting parallel group randomised trials. J Clin Epidemiol 2010; 63: 834-840.

23 Mousa SA, Mohamed S Inhibition of endothelial cell tube formation by the low molecular weight heparin, tinzaparin, is mediated by tissue factor pathway inhibitor. Thromb Haemost 2004; 92: 627-633.

24 Pfankuchen DB, Stolting DP, Schlesinger M, et al. Low molecular weight heparin tinzaparin antagonizes cisplatin resistance of ovarian cancer cells. Biochem Pharmacol 2015; 97: 147-157.

25 Feinstein AR, Sosin DM, Wells CK The Will Rogers phenomenon. Stage migration and new diagnostic techniques as a source of misleading statistics for survival in cancer. N Engl J Med 1985; 312: 1604-1608. 